\title{
Minimal extrathyroid extension in papillary micro carcinoma of the thyroid is an independent risk factor for relapse through lymph node and distant metastases
}

\author{
Robert Seifert $^{1,2,3}$, Michael Schäfers ${ }^{1,3,4}$, Barbara Heitplatz ${ }^{5}$, Laura Kerschke ${ }^{6}$, \\ Burkhard Riemann ${ }^{1,3^{*}}$ and Benjamin Noto ${ }^{1,3,7^{*}}$
}

1. Department of Nuclear Medicine, University Hospital Münster, Münster, Germany

2. Department of Nuclear Medicine, University Hospital Essen, Essen, Germany

3. West German Cancer Center (WTZ)

4. European Institute for Molecular Imaging, University of Münster, Münster, Germany.

5. Gerhard Domagk Institute of Pathology, University of Münster, Münster, Germany.

6. Institute of Biostatistics and Clinical Research, University of Münster, Münster, Germany

7. Department of Radiology, University Hospital Münster, Münster, Germany

*Both senior authors contributed equally

\section{Corresponding Author:}

Dr. med. Benjamin Noto

Department of Nuclear Medicine

University Hospital Münster

Albert-Schweitzer-Campus 1

D-48149 Münster, Germany

Tel: +49-251- 8347302

Fax: +49-251- 8349656

Benjamin.Noto@ukmuenster.de

ORCID ID: 0000-0002-1185-3709 
Short title: Minimal extrathyroid extension of microcarcinoma

Key words: thyroid; microcarcinoma; minimal extrathyroid extension.

\section{Word count: 4958}

\section{Disclosure:}

Funding: This study had no funding.

Conflict of Interest: The authors declare that they have no conflict of interest. 


\section{ABSTRACT}

\section{Aims}

Minimal extrathyroid extension (mETE) is no longer considered in the new $8^{\text {th }}$ edition of the AJCC/UICC staging system. Therefore, papillary thyroid microcarcinoma with mETE previously staged as pT3 will now be staged as pT1a and most likely not receive adjuvant radioiodine therapy. However, it remains unclear if mETE is associated with higher aggressiveness in papillary thyroid microcarcinoma. Therefore, the aim of this study was to investigate if mETE is associated with higher risk of lymph node or distant metastases.

\section{Methods}

721 patients with thyroid papillary microcarcinoma presenting at our department for postoperative counseling from $05 / 1983$ to $8 / 2012$ were included in this retrospective analysis (median follow-up time 9.30 years). The impact of mETE on the presence of lymph node metastases at thyroidectomy and relapse through lymph node and distant metastases was assessed by logistic regression and Fine-Gray model analyses.

\section{Results}

$10.7 \%(n=77)$ of patients had mETE. mETE was an independent risk factor for lymph node metastases at thyroidectomy with an adjusted odds ratio of $4.33(95 \% \mathrm{CI}: 2.02-9.60, \mathrm{p}<0.001)$ in multivariable analysis. Patients with mETE had significantly more relapses through lymph node (over 5 years: $13.1 \%$ vs. $1.25 \%$; $<<0.001$ ) and distant metastases (over 5 years: $7.8 \%$ vs. 1.1\%; $p$ $<0.001)$ compared to patients without mETE. mETE was an independent risk factor for relapse through lymph node and distant metastases in multivariable analysis (hazard ratio: 7.78, 95\%CI: 2.87-21.16, $\mathrm{p}<0.001$ and 4.09, 95\%CI: $1.25-13.36, \mathrm{p}=0.020)$. 


\section{Conclusion}

mETE is a statistically significant and independent risk factor for relapse through lymph node and distant metastases in papillary microcarcinoma. Therefore, future studies should evaluate, if patients with mETE and microcarcinoma might benefit from intensified surveillance and therapy.

\section{ABBREVIATIONS}

AJCC: American Joint Committee on Cancer

ATA: American thyroid association

DTC: Differentiated thyroid cancer

LNM: Lymph node metastases

METE: Minimal extrathyroid extension

PTC: Papillary thyroid cancer

PTMC: Papillary thyroid microcarcinoma

UICC: Union for International Cancer Control 


\section{INTRODUCTION}

Differentiated thyroid cancer (DTC) is classified according to the AJCC/UICC TNM system and has an increasing incidence, especially of small papillary tumors $\leq 10 \mathrm{~mm}(1)$. Up to the $7^{\text {th }}$ edition of the TNM system, minimal extrathyroid extension (mETE) was considered in determining the Tstage. However, several studies demonstrated mETE to have no impact on disease-related mortality, e.g. a study by Hay et al. with 3,524 patients or a meta-analysis by Diker-Cohen with 23,816 patients (2-6). Also, there are studies demonstrating no impact of mETE on recurrence free survival in DTC (7-9).

Since the TNM system strives for optimal prediction of cancer-related overall survival, the new $8^{\text {th }}$ edition of the AJVV/UICC cancer staging manual no longer considers mETE (10). In consequence, tumors $<4 \mathrm{~cm}$ with mETE which would have been classified as T3 according to the $7^{\text {th }}$ edition are now classified as $\mathrm{T} 1$ when $\leq 2 \mathrm{~cm}$ or T2 if $>2$ and $\leq 4 \mathrm{~cm}(11-13)$.

However, patients wth mETE in the aforementioned studies had significantly more often radioiodine therapy (RAI) than those without mETE. This is not surprising since according to the $7^{\text {th }}$ edition of TNM tumors with mETE were classified as at least T3, for which according to ATA and the European Society for Medical Oncology radioiodine therapy should be considered $(14,15)$. Hence, tumors with mETE could represent a more aggressive subset of tumors, only showing comparable survival rates to completely intrathyroidal tumors due to higher rates of initial radioiodine therapy according to the higher $\mathrm{T}$ stages.

Moreover, in the aforementioned studies, the impact of mETE was not studied in a microcarcinoma (PTMC) only group, but for tumors with various sizes. In the case of a large 4cm-sized tumor, it seems plausible that mETE might not additionally influence survival. Yet, in small tumors with a diameter $\leq 1 \mathrm{~cm}, \mathrm{mETE}$ might still be clinically relevant. The impact of 
removing mETE from T-staging in the $8^{\text {th }}$ edition of the TNM system is especially pronounced for papillary carcinoma $\leq 10 \mathrm{~mm}$ with $\mathrm{mETE}$, which will now be attributed the lowest possible T-stage pT1a instead of T3.

Therefore, the aim of the present study was to investigate, if mETE is an independent risk factor for tumor relapse in a large cohort of patients with PTMC and should be accounted for. 


\section{MATERIALS AND METHODS}

\section{Patients, Postoperative Management and Follow-up}

For this retrospective analysis, 721 consecutive patients with PTMC initially presenting in our department for postoperative counseling from $05 / 1983$ to $08 / 2012$ were enrolled. The median follow-up time was 9.30 years, with the last follow-up data recorded in 12/2020.

Extent of thyroidectomy and lymph node resection are detailed in Table 1. Histological and TNM classifications were present for all patients. To ensure consistent TNM classification, all patients were (re-)classified according to the AJCC/UICC $6^{\text {th }}$ edition (1997). Patients were divided into two groups: Tumors confined to the thyroid and those with mETE as previously published (16,17). Tumors were designated as mETE when the pathology report of the thyroidectomy sample stated minimal extrathyroid extension $(12,18) .74 .9 \%$ of patients received adjuvant RAI treatment

with consecutive ${ }^{131} \mathrm{I}$-whole-body scintigraphy after initial presentation. Between surgery and ${ }^{131} \mathrm{I}$ treatment, L-thyroxine treatment was withheld or stopped for $4-6$ weeks (19). In those patients that received RAI-therapy, stimulated thyroglobulin measurement, cervical ultrasound and diagnostic whole-body scintigraphy with ${ }^{131}$ I were performed 3 to 6 months and 1 year after initial adjuvant RAI in accordance with national and international standards prevailing at the time $(20,21)$. If one or more of these diagnostic tests were positive, further courses of ${ }^{131}$ I were given as needed (19). Long-term follow-up consisted of Tg measurement on LT4 therapy, serum thyrotropin and neck ultrasound yearly.

Table 1 describes the patients' characteristics in detail. The study protocol was approved by the local ethics committee (2019-459-f-S) and performed in accordance with the 1964 Declaration of Helsinki and its later amendments. 


\section{Endpoints}

Primary endpoints were cumulative incidence of lymph node metastases (1) and distant metastases relapse (2). Secondary endpoint was the presence of lymph node metastases in the thyroidectomy sample. Analyses were adjusted for extent of thyroidectomy and lymph node dissection if numerically feasable.

Distant metastases were diagnosed based on surgery with histologic workup or on a composite score requiring positive RAI imaging findings and elevated thyroglobulin ( $\mathrm{Tg}$ ) levels. Relapse through distant metastases was considered present when distant metastases were diagnosed after thyroidectomy, including recurrence in non-regional lymph nodes or visceral sites. Table 2 provides details on the detection of distant metastases. LNM-relapse was considered in two cases: 1) When LNM were diagnosed in patients without LNM in the thyroidectomy sample after thyroidectomy. 2) When LNM became apparent in patients who had LNM in the thyroidectomy sample in the course of follow-up after initial adjuvant RAI-treatment. In both cases diagnoss were based on a composite score consisting of RAI imaging and elevated thyroglobulin levels or surgery with histological work up. Presence of LNM in the thyroidectomy sample was histologically ascertained in all patients that underwent lymph node resection.

\section{Statistical Analysis}

Normally-distributed data are described using mean and standard deviation, non-normallydistributed using median and interquartile range. Normality was assessed by histograms and skewness statistics. Univariable and multivariable logistic regression analyses were carried out to evaluate the effect of age, sex, tumor size, multifocality, extent of lymph node resection and mETE on the presence of LNM at thyroidectomy, in the subgroup of patients that had lymph node 
resection (i.e. known nodal status at thyroidectomy). Results are reported as odds ratios (OR), corresponding $95 \%$ confidence intervals $(95 \% \mathrm{CI})$ and $\mathrm{p}$-values of the Wald test.

Time-to-event data were analyzed within a competing risk framework accounting for the competing risk of death. As distant metastases at thyroidectomy were considered a terminal event, the corresponding endpoint was analysed in the subgroup of patients without distant metastases at thyroidectomy. Cumulative incidences of distant metastases and LNM relapse after thyroidectomy were estimated based on the Fine-Gray model and compared between mETE positive and negative patients using Gray's test $(22,23)$. To adjust for further factors, a multivariable Fine-Gray subdistribution hazard regression was conducted. Results are presented as subdistribution hazard ratios (HR), corresponding 95\% CIs and p-values. Follow-up times were calculated by reverse Kaplan-Meier. All inferential statistics were intended to be exploratory and were interpreted accordingly. The reported two-sided p-values were used only to generate new hypotheses. p-values $\leq 0.05$ were considered statistically significant. Analyses were performed using $\mathrm{R}$ statistical software version 3.6.1 (The R Foundation, r-project.org). 


\section{RESULTS}

\section{Patient Characteristics}

Between 05/1983 and 28/2012, 721 patients with PTMC presented for postoperative counseling in our department. Patients were followed up on a yearly basis (median follow up time 9.30 years). No patient died due to thyroid cancer. mETE was present in $77(10.7 \%)$ of patients Patient characteristics are summarized in Table 1. Comparative characteristics between patients with confined tumors and mETE are presented in Table 3.

\section{Differences between Patients with and without mETE}

The proportion of patients with LNM in the thyroidectomy sample (subcohort of patients with lymph nodes resected at the thyroidectomy, N=216) was higher in patients with mETE $(27 / 44$, $61.4 \%)$ compared to those without $\mathrm{mETE}(49 / 172,28.5 \%, \mathrm{p}<0.001)$. LNM-relapse occurred in 11 out of 77 patients (14.3\%) with mETE in the course of follow-up and 10 out of 644 patients without mETE (1.6\%). Relapse through distant metastases (subcohort of patients without known distant metastases at thyroidectomy, $\mathrm{N}=719$ ) occurred in 7 out of 77 patients in the subgroup of patients with mETE (9.1\%), and in 8 out of 642 patients with confined tumors (1.2\%). See Table 3 for additional differences between patients with mETE and confined tumors. To adjust for interfering effects and loss to follow-up, multivariate regressions and survival analysis were performed in the next section.

\section{Risk Factors Associated with Lymph Node Metastases Detected at Thyroidectomy}

Risk factors associated with LNM detected at thyroidectomy where analyzed in the subset of patients that had lymph nodes removed at thyroidectomy $(n=216)$. In 140 out of those 216 
patients (64.8\%), no LNM were found, 55 (25.5\%) had central LNM and $21(9.7 \%)$ LNM in the lateral compartment.

The proportion of patients with LNM was higher in patients with mETE $(27 / 44,61.4 \%)$ compared to those without mETE $(49 / 172,30,2 \%, \mathrm{p}<0.001)$.

Results of the multivariable analysis using logistic regression are summarized in Table 4 and visualized in Figure 1. Sex, extent of lymph node resection (neck dissection vs. node picking) and mETE were significant risk factors for LNM, while tumor size was not. Adjusted odds ratio for LNM in regard to mETE was found to be 4.33 (95\%CI: 2.02-9.60, $\mathrm{p}<0.001)$.

\section{Risk Factors Associated with Lymph Node Metastases Relapse}

LNM relapse occurred in 21 out of 721 patients during the course of follow up (11/77 of patients with mETE, 10/644 without mETE). The five-year cumulative incidences were given by 13.12\% (95\% CI: 6.69-21.77) and 1.25\% (95\% CI: 0.59-2.37, p<0.001), for patients with mETE and those with confined tumors, respectively (Figure 2).

In the multivariable competing risk regression (Table 5), mETE (HR: 7.80, 95\%CI: 2.8721.16, $\mathrm{p}<0.001)$, male sex (HR: 4.17, 95\%CI: 1.63-10.67, $\mathrm{p}=0.003)$, and tumor size (HR: 1.15 , 95\%CI: 1.02-1.30, $\mathrm{p}=0.022$ ) were found to be independent risk factors for LNM relapse while age and multifocality were not.

\section{Risk Factors Associated with Distant Metastasis Relapse}

Distant metastases occurred in 15 out of 719 patients without distant metastases at thyroidectomy during the course of follow-up (mETE: 7/77, confined: 8/642). The five-year cumulative incidences were given by $7.79 \%$ (95\% CI: $3.16-15.19)$ and $1.11 \%$ (95\% CI: 0.50-2.19, $\mathrm{p}<0.001$ ), for patients with mETE and those with confined tumors respectively (Figure 3 ). 
In multivariable competing risk regression (Table 6) both mETE and the presence of LNM at thyroidectomy (N1 vs. N0) were found to be independent risk factors for distant metastases occurrence after thyroidectomy (HR: $4.09,95 \% \mathrm{CI}: 1.25-13.36, \mathrm{p}=0.020$ and $\mathrm{HR}: 8.76,95 \% \mathrm{CI}$ : $1.16-66.17, \mathrm{p}=0.035)$. 


\section{DISCUSSION}

The risk of lymph node metastases at thyroidectomy and relapse through LNM and distant metastases in patients with PTMC was analyzed with regard to mETE by the present study. mETE was found to be an independent risk factor for the presence of LNM at thyroidectomy and an independent risk factor for relapse through both distant and LNM.

The implications of mETE in papillary thyroid cancer are highly controversial. Multiple studies have reported that mETE is not associated with higher rates of LNM, distant metastases, or mortality in differentiated thyroid cancer $(3,4,24)$. Therefore, mETE was removed from the new $8^{\text {th }}$ edition of the TNM staging system. However, it seems unjustified to compare the outcome of patients with mETE to those without, as both groups have been treated with different intensity: patients with mETE significantly more often received RAI, compared to patients without mETE (2). Therefore, the not observed difference in outcome between the groups might have been caused by confounding factors.

The impact of removing mETE from $\mathrm{T}$ staging is especially pronounced for papillary carcinoma $\leq 10 \mathrm{~mm}$ with $\mathrm{mETE}$, which would have previously been staged as T3. With the new $8^{\text {th }}$ edition of TNM, these tumors are now attributed to the lowest possible stage pT1a. Very recent studies including differentiated thyroid cancer of all sizes indicate that not only gross, but also minimal extrathyroid extension is associated with increased mortality and recurrence $(25,26)$. To further corroborate these findings, the implications of mETE was assessed in a large, homogenous group of patients with PTMC by the present study.

Increased odds for LNM presence at surgery in patients with mETE were observed in the present study. This indicates that mETE tumors show a more aggressive phenotype. The finding is 
well in line with the results of Zhi et al. and others, finding mETE to be a risk factor for LNM in patients with PTMC (25-31).

Castagna et al. reported that mETE is a risk factor for LNM only in patients with a tumor size greater than $1.5 \mathrm{~cm}$ (32). This in contrast to our findings, which showed that mETE positive patients are more frequently affected by LNM at thyroidectomy than mETE negative patients, irrespective of tumor size (Figure 1).

The role of mETE for relapse through distant metastases in PTMC is likewise controversial. Six previous studies with PTMC-only cohorts could not find an impact of mETE on any cancer recurrence $(5,26,29,31,33,34)$. However, the cohorts in those studies were rather small, ranging from 144 to 288 patients. Three studies with larger PTMC-only cohorts, ranging from 287 to 531 patients, were able to demonstrated an impact of mETE on relapse in univariable analysis $(17,27,35)$. However, these studies did not investigate distant metastsases relapse, but any cancer recurrence. To date, there was no evidence for a higher rate of relapse for distant metastases associated with mETE. Our study in contrast, employing a cohort of 721 patients, could show for the first time that mETE is a statistically significant independent risk factor both for LNM and distant metastases relapse. As distant metastases are associated with a significantly worsened prognosis, this finding is of great clinical relevance (15).

The presence of LNM at thyroidectomy was identified as a further independent risk factor for relapse through distant metastases. Therefore, patients with LNM and mETE seem to have a particularly high risk of distant metastases after thyroidectomy compared to patients without both characteristics. Due to the delayed effects associated with mETE (i.e. relapse through distant metastases) it seems advisable to still integrate mETE in the T stage as proposed by Schmid et al. (12). 
Given the higher cumulative incidence of LNM and distant metastases relapse in patients with PTMC and mETE compared to those without, adjuvant RAI might be advisable. A study by Rosario et al. investigated disease recurrence rates of patients with mETE who did not receive adjuvant RAI (36). Only $2 \%$ of their patients had recurrent disease, leading to their conclusion that RAI can be omitted in patients with mETE. However, only patients without LNM at diagnosis and only 20 patients with PTMC have been enrolled, which hampers their results' transferability. As mETE is a risk factor for lymph node and especially distant metastases, mETE-positive tumors might be biologically more aggressive and could deserve an intensified treatment. Especially patients with both LNM in the thyroidectomy sample and mETE might benefit from an initial RAI. Until further studies explicitly investigate the benefit of RAI in patients with PTMC and mETE, the clinician has to carefully review the pathological report after thyroidectomy and discuss the option of adjuvant RAI when mETE is present.

The present study faces some limitations. It was conducted retrospectively and might therefore be influenced by selection biases. To counteract this effect, all patients from a period larger than 30 years have been included. However, the accuracy of diagnostic procedures has increased, which could influence the detection of metastases. Despite the long recruitment period, the number of included patients is relatively small. The presence of LNM at thyroidectomy could only be evaluated in patients that underwent lymph node resection. These patients might not represent a random subset of PTMC patients, which could limit our findings regarding this endpoint. The extent of thyroidectomy could not be included into the competing risk models of lymph node and distant metastases recurrence due to numerical reasons (nearly all patients had total thyroidectomy). Given the low incidence of mETE, multicentric analyses have to corroborate the present initial findings. Another limitation arises from the controversy among pathologists about what constitutes mETE, with currently no standardized histopathologic criteria $(18,37)$. The 
pathology reports from which the presence of mETE was derived for this study were issued by numerous pathologists from different institutions, possibly applying varying criteria for the diagnosis of mETE.

\section{CONCLUSION}

Minimal extrathyroid extension is an independent risk factor for cancer relapse through lymph node and distant metastases in papillary microcarcinoma of the thyroid. Therefore, future studies should evaluate, if patients with $\mathrm{mETE}$ and microcarcinoma might benefit from intensified surveillance and/- or therapy.

\section{ACKNOWLEDGMENTS}

The graphical absctract was created with BioRender.com 


\section{KEY POINTS}

QUESTION: Is minimal extrathyroid extension an independent risk factor for cancer relapse in papillary microcarcinoma of the thyroid?

PERTINENT FINDINGS: This retrospective cohort study included 721 patients with thyroid papillary microcarcinoma presenting for postoperative counseling in a single institution with a median follow-up time of 9.30 years. Minimal extrathyroid extension was found to be a statistically significant and independent risk factor of cancer relapse through both lymph node and distant metastases.

IMPLICATIONS FOR PATIENT CARE: Papillary microcarcinoma patients with minimal extrathyroid extension might benefit from intensified surveillance and/ or therapy. 


\section{REFERENCES}

1. Davies L, Welch HG. Increasing incidence of thyroid cancer in the United States, 1973-2002. JAMA. 2006;295:2164-2167.

2. Diker-Cohen T, Hirsch D, Shimon I, et al. Impact of minimal extra-thyroid extension in differentiated thyroid cancer: Systematic Review and Meta-analysis. J Clin Endocrinol Metab. 2018

3. Ito $\mathrm{Y}$, Tomoda $\mathrm{C}$, Uruno $\mathrm{T}$, et al. Minimal extrathyroid extension does not affect the relapsefree survival of patients with papillary thyroid carcinoma measuring $4 \mathrm{~cm}$ or less over the age of 45 years. Surg Today. 2006;36:12-18.

4. Hay ID, Johnson TR, Thompson GB, Sebo TJ, Reinalda MS. Minimal extrathyroid extension in papillary thyroid carcinoma does not result in increased rates of either cause-specific mortality or postoperative tumor recurrence. Surgery. 2016;159:11-19.

5. Woo CG, Sung CO, Choi YM, et al. Clinicopathological significance of minimal extrathyroid extension in solitary papillary thyroid carcinomas. Ann Surg Oncol. 2015;22 Suppl 3:S728-33.

6. Al-Qurayshi Z, Shama MA, Randolph GW, Kandil E. Minimal extrathyroidal extension does not affect survival of well-differentiated thyroid cancer. Endocr Relat Cancer. 2017;24:221226.

7. Nixon IJ, Ganly I, Patel S, et al. The impact of microscopic extrathyroid extension on outcome in patients with clinical T1 and T2 well-differentiated thyroid cancer. Surgery. 2011;150:12421249.

8. Shin JH, Ha TK, Park HK, et al. Implication of minimal extrathyroidal extension as a prognostic factor in papillary thyroid carcinoma. Int J Surg. 2013;11:944-947. 
9. Jin BJ, Kim MK, Ji YB, Song CM, Park JH, Tae K. Characteristics and significance of minimal and maximal extrathyroidal extension in papillary thyroid carcinoma. Oral Oncol. $2015 ; 51: 759-763$.

10. Tuttle RM, Haugen B, Perrier ND. Updated American Joint Committee on Cancer/TumorNode-Metastasis staging system for differentiated and anaplastic thyroid cancer (Eighth Edition): What changed and why? Thyroid. 2017;27:751-756.

11. Amin MB, Greene FL, Edge SB, eds. AJCC cancer staging manual. 8th edition. Schweiz, Chicago, IL: Springer; AJCC American Joint Committee on Cancer; 2017.

12. Schmid KW, Synoracki S, Dralle H, Wittekind C. Vorschlag zu einer erweiterten pTNMKlassifikation der Schilddrüsenkarzinome : Kommentar zu Defiziten der 8. Auflage der TNMKlassifikation. Pathologe. 2018;39:49-56.

13. Amin MB, Greene FL, Edge SB, et al. The eighth edition AJCC cancer staging manual: Continuing to build a bridge from a population-based to a more "personalized" approach to cancer staging. CA Cancer J Clin. 2017;67:93-99.

14. Filetti S, Durante C, Hartl D, et al. Thyroid cancer: ESMO Clinical Practice Guidelines for diagnosis, treatment and follow-up†. Ann Oncol. 2019;30:1856-1883.

15. Haugen BR, Alexander EK, Bible KC, et al. 2015 American Thyroid Association management guidelines for adult patients with thyroid nodules and differentiated thyroid cancer: The American Thyroid Association guidelines task force on thyroid nodules and differentiated thyroid cancer. Thyroid. 2016;26:1-133.

16. Youngwirth LM, Adam MA, Scheri RP, Roman SA, Sosa JA. Extrathyroidal extension is associated with compromised survival in patients with thyroid cancer. Thyroid. 2017;27:626631. 
17. Mercante G, Frasoldati A, Pedroni C, et al. Prognostic factors affecting neck lymph node recurrence and distant metastasis in papillary microcarcinoma of the thyroid: results of a study in 445 patients. Thyroid. 2009;19:707-716.

18. Mete O, Rotstein L, Asa SL. Controversies in thyroid pathology: thyroid capsule invasion and extrathyroidal extension. Ann Surg Oncol. 2010;17:386-391.

19. Vrachimis A, Riemann B, Mäder U, Reiners C, Verburg FA. Endogenous TSH levels at the time of 131I ablation do not influence ablation success, recurrence-free survival or differentiated thyroid cancer-related mortality. Eur J Nucl Med Mol Imaging. 2016;43:224-231.

20. Luster M, Clarke SE, Dietlein M, et al. Guidelines for radioiodine therapy of differentiated thyroid cancer. Eur J Nucl Med Mol Imaging. 2008;35:1941-1959.

21. M. Dietlein, W. Eschner, F. Grünwald, M. Lassmann, F.A. Verburg, M. Luster. DGNHandlungsempfehlung (S1-Leitlinie) Radioiodtherapie beim differenzierten Schilddrüsenkarzinom. https://www.nuklearmedizin.de/leistungen/leitlinien/docs/0310021_S1_Radioiodtherapie_differenziertes_Schilddruesenkarziom_2015-10.pdf Accessed on $2021 / 02 / 26$

22. Fine JP, Gray RJ. A proportional hazards model for the subdistribution of a competing risk. $J$ Am Stat Assoc. 1999;94:496-509.

23. Gray RJ. A class of K-Sample Tests for comparing the cumulative incidence of a competing risk. The Annals of Statistics. 1988;16:1141-1154.

24. Ito Y, Tomoda C, Uruno T, et al. Prognostic significance of extrathyroid extension of papillary thyroid carcinoma: massive but not minimal extension affects the relapse-free survival. World J Surg. 2006;30:780-786. 
25. Liu Z, Huang Y, Chen S, et al. Minimal extrathyroidal extension affects the prognosis of differentiated thyroid cancer: Is there a need for change in the AJCC classification system? PLoS ONE. 2019;14:e218171.

26. Danilovic DLS, Castroneves LA, Suemoto CK, et al. Is there a difference between minimal and gross extension into the strap muscles for the risk of recurrence in papillary thyroid carcinomas? Thyroid. 2020.

27. Lombardi CP, Bellantone R, Crea C de, et al. Papillary thyroid microcarcinoma: extrathyroidal extension, lymph node metastases, and risk factors for recurrence in a high prevalence of goiter area. World J Surg. 2010;34:1214-1221.

28. Zhi J, Zhao J, Gao M, et al. Impact of major different variants of papillary thyroid microcarcinoma on the clinicopathological characteristics: the study of 1041 cases. Int J Clin Oncol. 2018;23:59-65.

29. Moon HJ, Kim E-K, Chung WY, Yoon JH, Kwak JY. Minimal extrathyroidal extension in patients with papillary thyroid microcarcinoma: is it a real prognostic factor? Ann Surg Oncol. 2011;18:1916-1923.

30. Ahmaddy F, Wenter V, Ilhan H, et al. Effects of the minimal extrathyroidal extension on early response rates after (adjuvant) initial radioactive iodine therapy in PTC patients. Cancers (Basel). 2020;12.

31. Siddiqui S, White MG, Antic T, et al. Clinical and pathologic predictors of lymph node metastasis and recurrence in papillary thyroid microcarcinoma. Thyroid. 2016;26:807-815.

32. Castagna MG, Forleo R, Maino F, et al. Small papillary thyroid carcinoma with minimal extrathyroidal extension should be managed as ATA low-risk tumor. J Endocrinol Invest. 2018;41:1029-1035. 
33. Besic N, Pilko G, Petric R, Hocevar M, Zgajnar J. Papillary thyroid microcarcinoma: prognostic factors and treatment. $J$ Surg Oncol. 2008;97:221-225.

34. Chow S-M, Law SCK, Chan JKC, Au S-K, Yau S, Lau W-H. Papillary microcarcinoma of the thyroid-Prognostic significance of lymph node metastasis and multifocality. Cancer. 2003;98:31-40.

35. Tran B, Roshan D, Abraham E, et al. An analysis of the American Joint Committee on Cancer 8th Edition T Staging System for papillary thyroid carcinoma. J Clin Endocrinol Metab. 2018;103:2199-2206.

36. Rosario PW, Mourão G, Calsolari MR. Risk of recurrence in patients with papillary thyroid carcinoma and minimal extrathyroidal extension not treated with radioiodine. $J$ Endocrinol Invest. 2019;42:687-692.

37. Su HK, Wenig BM, Haser GC, et al. Inter-observer variation in the pathologic identification of minimal extrathyroidal extension in papillary thyroid carcinoma. Thyroid. 2016;26:512-517. 


\section{TABLES}

Table 1: Patient characteristics of the included cohort.

\begin{tabular}{|c|c|c|c|c|}
\hline Patient characteristics & Total cohort & Confined & mETE & $\begin{array}{c}\text { Uni-variate } \\
\text { Analysis } \\
\text { p* }^{*}\end{array}$ \\
\hline Number of patients(\%) & 721 & $644(89.3)$ & $77(10.7)$ & \\
\hline Sex $=$ male $($ number $(\%))$ & $154(21.4)$ & $145(22.5)$ & $9(11.7)$ & 0.041 \\
\hline Age in years (mean(SD)) & $47.95(12.83)$ & $48.02(12.77)$ & $47.38(13.42)$ & 0.678 \\
\hline $\begin{array}{l}\text { Median follow-up in years } \\
(95 \% \mathrm{CI})^{* *}\end{array}$ & $9.30(8.89-9.94)$ & $8.94(8.40-9.43)$ & $\begin{array}{c}12.85(10.76- \\
14.38)\end{array}$ & 0.002 \\
\hline Extent of thyroidectomy(\%) & & & & $<0.001$ \\
\hline Hemithyroidectomy & $18(2.5)$ & $18(2.8)$ & $0(0.0)$ & \\
\hline Subtotal thyroidectomy & $126(17.5)$ & $125(19.4)$ & $1(1.3)$ & \\
\hline Total thyroidectomy & $577(80.0)$ & $501(77.8)$ & $76(98.7)$ & \\
\hline Lymph node dissection(\%) & & & & $<0.001$ \\
\hline No lymph nodes resected & $505(70.0)$ & $472(73.3)$ & $33(42.9)$ & \\
\hline Node picking & $51(7.1)$ & $46(7.1)$ & $5(6.5)$ & \\
\hline Central compartment node dissection & $120(16.6)$ & $93(14.4)$ & $27(35.1)$ & \\
\hline $\begin{array}{l}\text { Central and lateral neck node } \\
\text { dissection }\end{array}$ & $45(6.2)$ & $33(5.1)$ & $12(15.6)$ & \\
\hline $\begin{array}{l}\text { Number of removed lymph nodes } \\
\text { (median[IQR]) }\end{array}$ & $0[0,1]$ & $0[0,0]$ & $2[0,10]$ & $<0.001$ \\
\hline Adjuvant RAI treatment(\%) & $540(74.9)$ & $463(71.9)$ & $77(100.0)$ & $<0.001$ \\
\hline $\begin{array}{l}\text { Cumulative RAI activity in GBq } \\
\text { (median[IQR]) }\end{array}$ & $3.00[0.00,6.00]$ & $3.00[0.00,4.00]$ & $\begin{array}{c}6.00 \\
{[3.00,10.00]}\end{array}$ & $<0.001$ \\
\hline $\begin{array}{l}\text { Number of RAI treatments } \\
\text { (median[IQR]) }\end{array}$ & $1.00[0.00,2.00]$ & $1.00[0.00,1.00]$ & $\begin{array}{c}1.00 \\
{[1.00,2.00]}\end{array}$ & $<0.001$ \\
\hline $\begin{array}{l}\text { Pre RAI treatment TSH in } \mu \mathrm{U} / \mathrm{ml} \\
\text { (median[IQR]) }\end{array}$ & $43.06[15.62,77.26]$ & $\begin{array}{c}39.17 \\
{[13.24,76.89]}\end{array}$ & $\begin{array}{c}59.36 \\
{[42.20,77.72]}\end{array}$ & 0.001 \\
\hline $\begin{array}{l}\text { Pre RAI treatment thyreoglobulin } \\
\text { in } \mathrm{ng} / \mathrm{ml} \text { (median[IQR]) }\end{array}$ & $2.30[0.62,8.45]$ & $2.50[0.60,8.67]$ & $\begin{array}{c}2.20 \\
{[0.80,7.00]} \\
\end{array}$ & 0.935 \\
\hline
\end{tabular}

$\mathrm{RAI}=$ radioactive iodine; $\mathrm{SD}=$ standard deviation; $\mathrm{IQR}=$ inter quartile range. *Determined with Fisher's exact test for categorical variables and Wilcoxon test for continuous variables; $* *$ calculated by reverse Kaplan-Meier 
Table 2: Detailed report on patients with distant metastases.

\begin{tabular}{|c|c|c|c|c|c|}
\hline mETE & $\begin{array}{c}\text { Age at } \\
\text { initial } \\
\text { presentati } \\
\text { on }\end{array}$ & $\begin{array}{l}\text { Type of distant } \\
\text { metastasis }\end{array}$ & $\begin{array}{l}\text { Initial } \\
\text { LNM }\end{array}$ & $\begin{array}{l}\text { Tumor } \\
\text { diameter } \\
\text { in } \mathrm{mm}\end{array}$ & $\begin{array}{c}\text { Days between } \\
\text { postoperative } \\
\text { counseling and } \\
\text { confirmation of } \\
\text { metastasis }\end{array}$ \\
\hline No & 65 & Mediastinal LNM & No & 4 & 119 \\
\hline Yes & 68 & Bone & No & 5 & 4213 \\
\hline No & 69 & Bone & No & 1 & $-25^{*}$ \\
\hline No & 30 & Pulmonary & No & 7 & 454 \\
\hline No & 26 & Pulmonary & No & 10 & 830 \\
\hline No & 48 & Bone & No & 4 & 7 \\
\hline No & 25 & Bone & No & 9 & 0 \\
\hline No & 44 & Pulmonary & No & 7 & 40 \\
\hline Yes & 54 & $\begin{array}{l}\text { Bone and } \\
\text { Pulmonary }\end{array}$ & Yes & 9 & 50 \\
\hline Yes & 58 & Pulmonary & No & 9 & 121 \\
\hline Yes & 55 & Pulmonary & Yes & 5 & 61 \\
\hline No & 48 & Pulmonary & Yes & 4 & 4309 \\
\hline No & 15 & Pulmonary & Yes & 10 & 14 \\
\hline Yes & 15 & Pulmonary & Yes & 8 & 28 \\
\hline Yes & 37 & Pulmonary & Yes & 3 & 256 \\
\hline Yes & 23 & Pulmonary & Yes & 5 & 442 \\
\hline No & 6 & Pulmonary & Yes & 10 & 22 \\
\hline
\end{tabular}

* Initial diagnosis of thyroid cancer was made after bone metastasis confirmation. 
Table 3: Characteristics of patients with and without mETE.

\begin{tabular}{|c|c|c|c|c|}
\hline Patient characteristics & Total cohort & Confined & mETE & $\begin{array}{c}\text { Univariable } \\
\text { Analysis } \\
\text { p }^{*}\end{array}$ \\
\hline Number of patients $(\%)$ & 721 & $\begin{array}{c}644 \\
(89.3)\end{array}$ & $\begin{array}{c}77 \\
(10.7)\end{array}$ & \\
\hline $\begin{array}{l}\text { Number of metastasized lymph nodes } \\
\text { (median[IQR]) }\end{array}$ & $\begin{array}{c}0 \\
{[0,0]}\end{array}$ & $\begin{array}{c}0 \\
{[0,0]}\end{array}$ & $\begin{array}{c}0 \\
{[0,1]}\end{array}$ & $<0.001$ \\
\hline $\begin{array}{l}\text { Nodal stage given by histopathologic } \\
\text { examination of the surgical specimen(\%) }\end{array}$ & & & & $<0.001$ \\
\hline pN0 & $137(19.0)$ & $120(18.6)$ & $17(22.1)$ & \\
\hline pN1a & $57(7.9)$ & $39(6.1)$ & $18(23.4)$ & \\
\hline pN1b & $22(3.1)$ & $13(2.0)$ & $9(11.7)$ & \\
\hline $\mathrm{pNx}$ & $505(70.0)$ & $472(73.3)$ & $33(42.9)$ & \\
\hline $\begin{array}{l}\text { Five-year cumulative incidence of lymph } \\
\text { node metastases relapse in } \%(95 \% \mathrm{CI})\end{array}$ & $\begin{array}{c}2.54 \\
(1.57-3.91)\end{array}$ & $\begin{array}{c}1.25 \\
(0.59-2.37)\end{array}$ & $\begin{array}{c}13.12 \\
(6.69-21.77)\end{array}$ & $<0.001$ \\
\hline $\begin{array}{l}\text { Five-year cumulative incidence distant } \\
\text { metastases relapse in } \%(95 \% \mathrm{CI})^{* *}\end{array}$ & $\begin{array}{c}1.83 \\
(1.03-3.03)\end{array}$ & $\begin{array}{c}1.11 \\
(0.50-2.19)\end{array}$ & $\begin{array}{c}7.79 \\
(3.16-15.19)\end{array}$ & $<0.001$ \\
\hline Tumor size in mm (median[IQR] & $\begin{array}{c}5.00 \\
{[3.00,8.00]}\end{array}$ & $\begin{array}{c}5.00 \\
{[3.00,8.00]}\end{array}$ & $\begin{array}{c}8.00 \\
{[6.00,10.00]}\end{array}$ & $<0.001$ \\
\hline Multifocal disease(\%) & $135(18.7)$ & $118(18.3)$ & $17(22.1)$ & 0.520 \\
\hline
\end{tabular}


Table 4: Univariable and multivariable logistic regression analysis of risk factors for lymph node metastasis diagnosed in histologic workup of thyroidectomy sample adjusted for lymph node and thyroidectomy extent. Only the subgroup of patients that had lymph nodes resected are included $(\mathrm{N}=216)$.

\begin{tabular}{|c|c|c|c|c|c|c|}
\hline & LNM - & LNM + & $\begin{array}{c}\text { Odds ratios } \\
\text { univariable } \\
\text { analysis } \\
(95 \% \mathrm{CI})\end{array}$ & $\begin{array}{c}p \\
\text { univariable } \\
\text { analysis }\end{array}$ & $\begin{array}{c}\text { Odds ratios } \\
\text { multivariable } \\
\text { analysis } \\
(95 \% \mathrm{CI})\end{array}$ & $\begin{array}{c}p \\
\text { multivariable } \\
\text { analysis }\end{array}$ \\
\hline $\mathrm{N}$ & 140 & 76 & & & & \\
\hline Sex $=$ male $(\%)$ & $\begin{array}{c}24 \\
(17.1)\end{array}$ & $\begin{array}{c}25 \\
(32.9)\end{array}$ & $\begin{array}{c}2.37 \\
(1.24-4.56)\end{array}$ & 0.009 & $\begin{array}{c}2.83 \\
(1.36-5.98)\end{array}$ & 0.006 \\
\hline $\begin{array}{l}\text { Age } \\
(\text { mean }(\mathrm{SD}))\end{array}$ & $\begin{array}{c}44.79 \\
(12.50)\end{array}$ & $\begin{array}{c}41.54 \\
(13.92)\end{array}$ & $\begin{array}{c}0.98 \\
(0.96-1.00)\end{array}$ & 0.082 & $\begin{array}{c}0.98 \\
(0.96-1.01)\end{array}$ & 0.183 \\
\hline $\begin{array}{l}\text { Tumor size in mm } \\
\text { (median [IQR]) }\end{array}$ & $\begin{array}{c}7.00 \\
{[4.00,9.00]}\end{array}$ & $\begin{array}{c}7.00 \\
{[5.00,9.00]}\end{array}$ & $\begin{array}{c}1.05 \\
(0.94-1.18)\end{array}$ & 0.378 & $\begin{array}{c}1.04 \\
(0.91-1.18)\end{array}$ & 0.579 \\
\hline $\begin{array}{l}\text { Multifocality } \\
(\%)\end{array}$ & $\begin{array}{c}27 \\
(19.3)\end{array}$ & $\begin{array}{c}16 \\
(21.1)\end{array}$ & $\begin{array}{c}1.12 \\
(0.55-2.21)\end{array}$ & 0.756 & $\begin{array}{c}1.09 \\
(0.49-2.39)\end{array}$ & 0.834 \\
\hline $\begin{array}{l}\text { Neck dissection vs. node } \\
\text { picking }(\%)\end{array}$ & $\begin{array}{c}93 \\
(66.4)\end{array}$ & $\begin{array}{c}72 \\
(94.7)\end{array}$ & $\begin{array}{c}9.10 \\
(3.50-31.17)\end{array}$ & $<0.001$ & $\begin{array}{c}6.55 \\
(2.41-23.05)\end{array}$ & $<0.001$ \\
\hline $\begin{array}{l}\text { Total thyroidectomy vs. } \\
\text { subtotal/hemthyroidectomy } \\
\text { ( \%) }\end{array}$ & $\begin{array}{c}131 \\
(93.6)\end{array}$ & $\begin{array}{c}74 \\
(97.4)\end{array}$ & $\begin{array}{c}2.54 \\
(0.63-16.96)\end{array}$ & 0.241 & $\begin{array}{c}1.28 \\
(0.25-9.94)\end{array}$ & 0.782 \\
\hline mETE (\%) & $\begin{array}{c}17 \\
(12.1)\end{array}$ & $\begin{array}{c}27 \\
(35.5)\end{array}$ & $\begin{array}{c}3.99 \\
(2.02-8.09)\end{array}$ & $<0.001$ & $\begin{array}{c}4.33 \\
(2.02-9.60)\end{array}$ & $<0.001$ \\
\hline
\end{tabular}

$\mathrm{mETE}=$ minimal extrathyroid extension, $\mathrm{LNM}=$ lymph node metastases, $\mathrm{SD}=$ standard deviation; $\mathrm{IQR}=$ inter quartile range, $\mathrm{CI}=$ confidence interval. 
Table 5: Multivariable competing risk regression analysis for lymph node metastasis relapse after thyroidectomy based on Fine-Gray proportional subdistribution hazards model.

\begin{tabular}{lccc} 
Variable & HR & $\mathbf{9 5 \%}$ CI & p-value \\
\hline Sex (male-female) & 4.17 & $1.63-10.67$ & 0.003 \\
Age & 1.00 & $0.98-1.03$ & 0.690 \\
Tumor size & 1.15 & $1.02-1.30$ & 0.022 \\
Multifocality (yes-no) & 2.44 & $0.97-6.17$ & 0.060 \\
$\begin{array}{l}\text { Lymph nodes resected at } \\
\text { thyroidectomy (yes vs. no) }\end{array}$ & 3.23 & $1.33-7.86$ & 0.010 \\
& & & \\
mETE (yes-no) & 7.80 & $2.87-21.16$ & $<0.001$ \\
\hline
\end{tabular}

$\mathrm{HR}=$ hazard ratio, $\mathrm{CI}=$ confidence interval 
Table 6: Multivariable competing risk regression analysis for distant metastasis relapse after thyroidectomy based on Fine-Gray proportional subdistribution hazards model.

\begin{tabular}{lccc} 
Variable & HR & $\mathbf{9 5 \%}$ CI & p-value \\
\hline Sex (male-female) & 1.52 & $0.48-4.82$ & 0.470 \\
Age & 0.96 & $0.91-1.01$ & 0.140 \\
$\begin{array}{l}\text { Tumor size } \\
\text { Multifocality (yes-no) }\end{array}$ & 1.06 & $0.85-1.32$ & 0.610 \\
$\begin{array}{l}\text { N status at thyroidectomy (Nx } \\
\text { vs. N0) }\end{array}$ & 2.25 & $0.73-6.90$ & 0.160 \\
$\begin{array}{l}\text { N status at thyroidectomy (N1 } \\
\text { vs. N0) }\end{array}$ & 8.76 & $0.30-22.51$ & 0.390 \\
$\begin{array}{l}\text { mETE (yes-no) } \\
\text { NR }\end{array}$ & & & \\
\hline
\end{tabular}

$\mathrm{HR}=$ hazard ratio, $\mathrm{CI}=$ confidence interval 


\section{FIGURES}

Figure 1: Addjusted odds rations for lymph node metastases at thyroidectomy (evaluated in the subgroup of patients that had lymph nodes resected at thyroidectomy, $\mathrm{N}=216$ ). Odds ratios in tabularly form are found in Table 4.

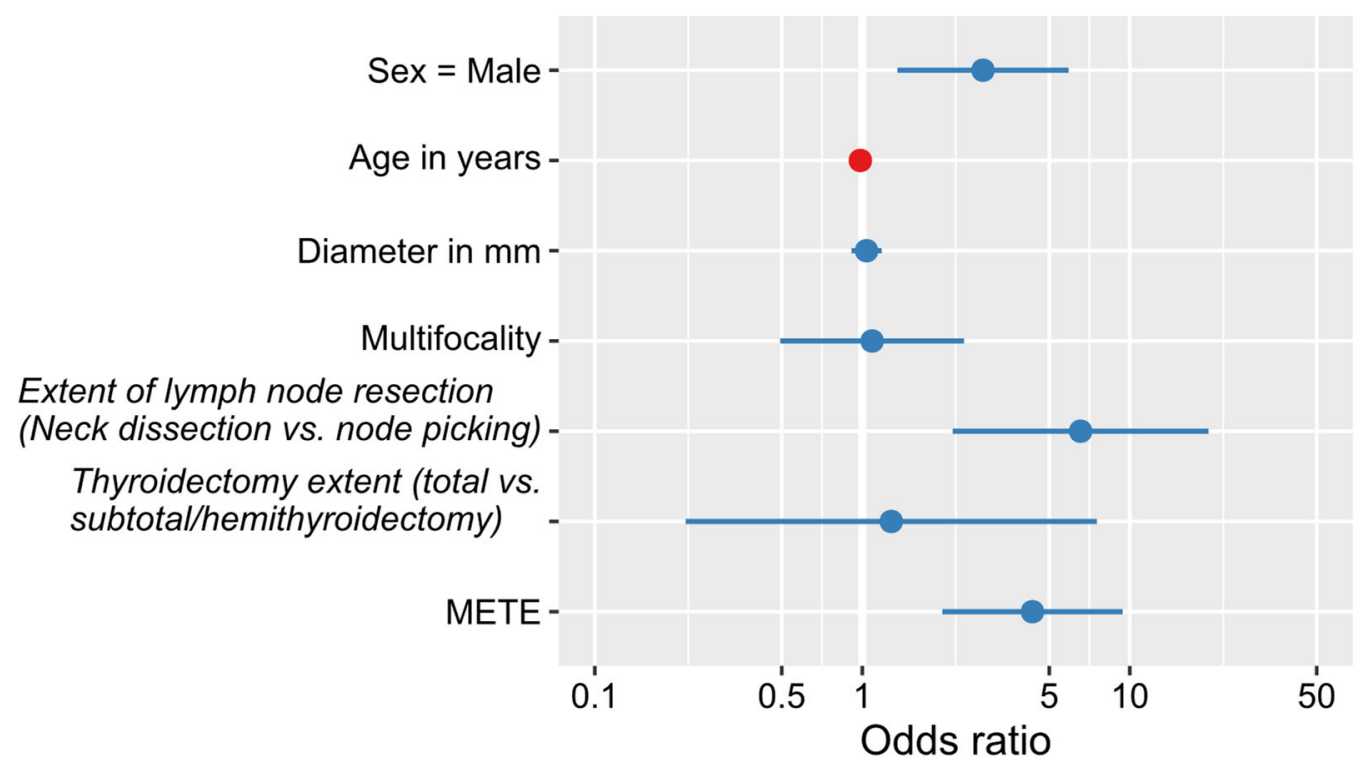


Figure 2: Cumulative incidence function of LNM-relapse after thyroidectomy stratified by mETE. Curves were compared using Gray's test $(\mathrm{N}=721)$.

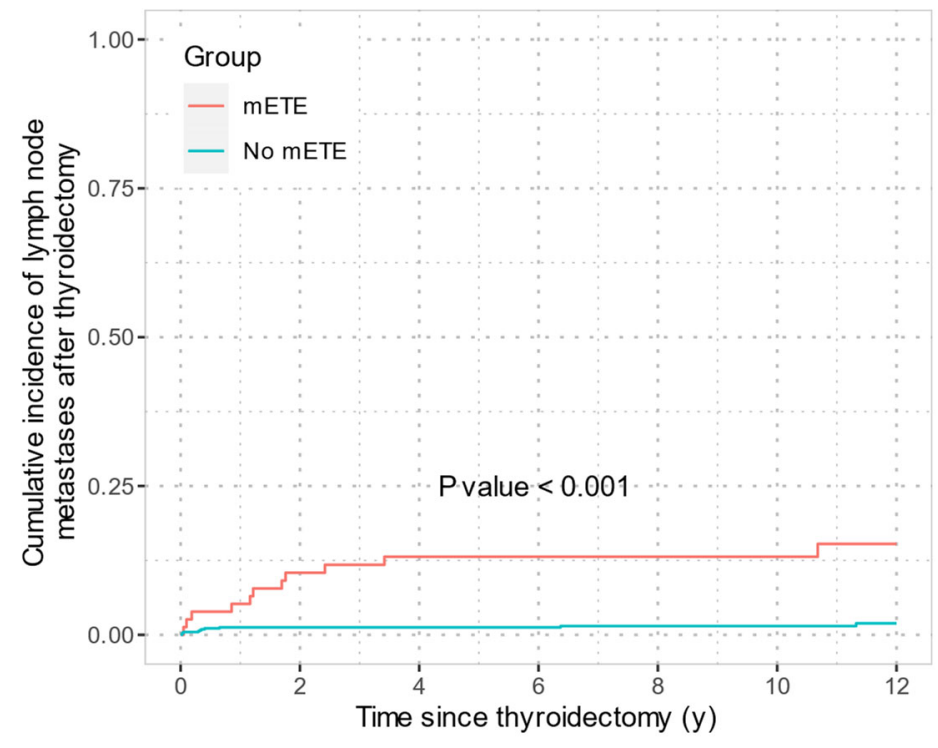

Figure 3: Cumulative incidence function of distant metastases relapse after thyroidectomy in the group of patients without distant metastases at thyroidectomy $(\mathrm{N}=719)$ stratified by mETE. Curves were compared using Gray's test.

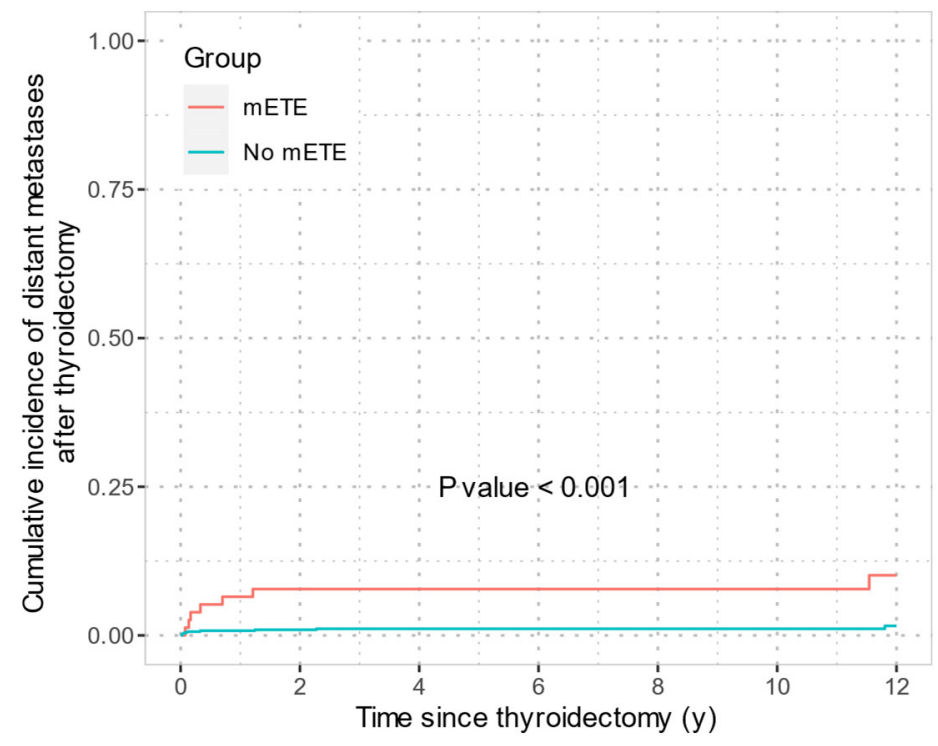




\section{GRAPHICAL ABSTRACT}

\section{Papillary microcarcinoma}

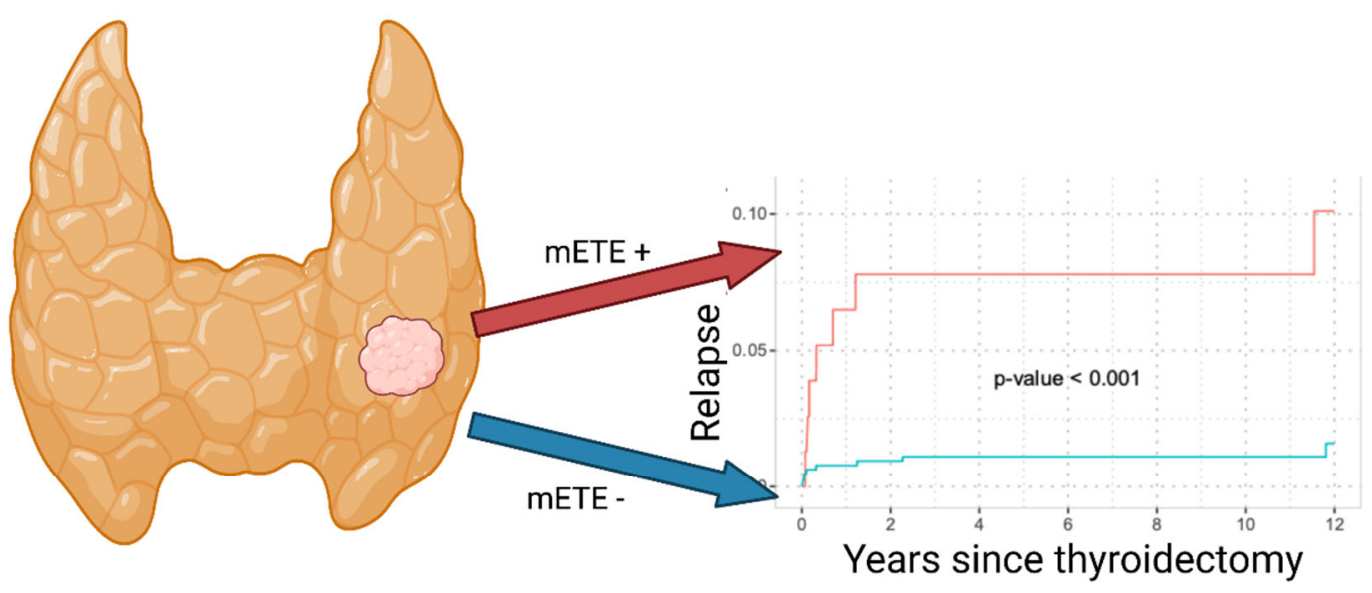

